\title{
New adjuvants for accelerated and enhanced antibody response
}

\author{
Immunization of animals and birds for the purposes of developing hyperimmune sera or purification \\ of specific antibodies heavily relies on adjuvants. PickCell Laboratories offers a new generation of \\ adjuvants that greatly accelerate development of specific response, decrease the required dose of \\ antigen and increase up to 100 -fold the concentration of specific antibody in serum.
}

Production of commercial neutralizing antibodies or specific antibodies for biological research relies heavily on adjuvants-compounds that may have different biological roles but all result in increase of either humoral or cytotoxic immune response. One should discriminate between local adjuvants and so-called central action adjuvants. Whereas the first group of compounds changes the properties of an antigen (creating a depot of antigen, improving antigen delivery to antigen-presenting cells or attracting effector cells to the site of injection), the second group affects the recipient of the antigen by increasing its immunocompetence, which can be defined as the ability to give strong immune response to presented antigen and is related to the number of and the state of activity of immune cells participating in the desired type of immune response.

Short peptides, which often originate from protein processing in tissue, may act as immunomodulators by changing the number and activity of immune cells and thus affect (activate or suppress) the ability of the antigen recipient to develop either humoral or cytotoxic immune response ${ }^{1}$. On the basis of short peptide activating motifs that have been discovered to affect the immune system ${ }^{2}$, we have developed a family of short synthetic peptides with immunomodulating properties. These artificial peptides, with a maximum size of eight amino acids, have demonstrated the properties of both local and central action adjuvants, thus affecting the general immunocompetence of the recipient as well as promoting immune response at the site of injection. One of them, named IMMACCEL-RTM, showed advantageous properties and excellent performance in accelerating immune response.

\section{Main properties of IMMACCEL-R}

IMMACCEL-R, when injected into the recipient animal, stimulates induction of cytokine cascades that closely resemble those seen at the early stages of inflammatory response, thus making the animal more

\section{Sergey Litvinov}

PickCell Laboratories BV, Lelystad, The Netherlands. Correspondence should be addressed to S.L. (slitvinov@brd-corp.com). prone to give a strong immune response when injected with the antigen. Among the genes affected are those encoding main cytokines and their receptors, as well as several chemokines typical of the inflammatory response (Fig. 1). When mixed with antigen and delivered together at the site of injection (intradermal or subcutaneous), IMMACCEL-R further improves the immune response by enhancing presentation of processed antigen by dendritic cells (data not shown) and increasing the specific proliferation of T-helper or T-killer cells, depending on the

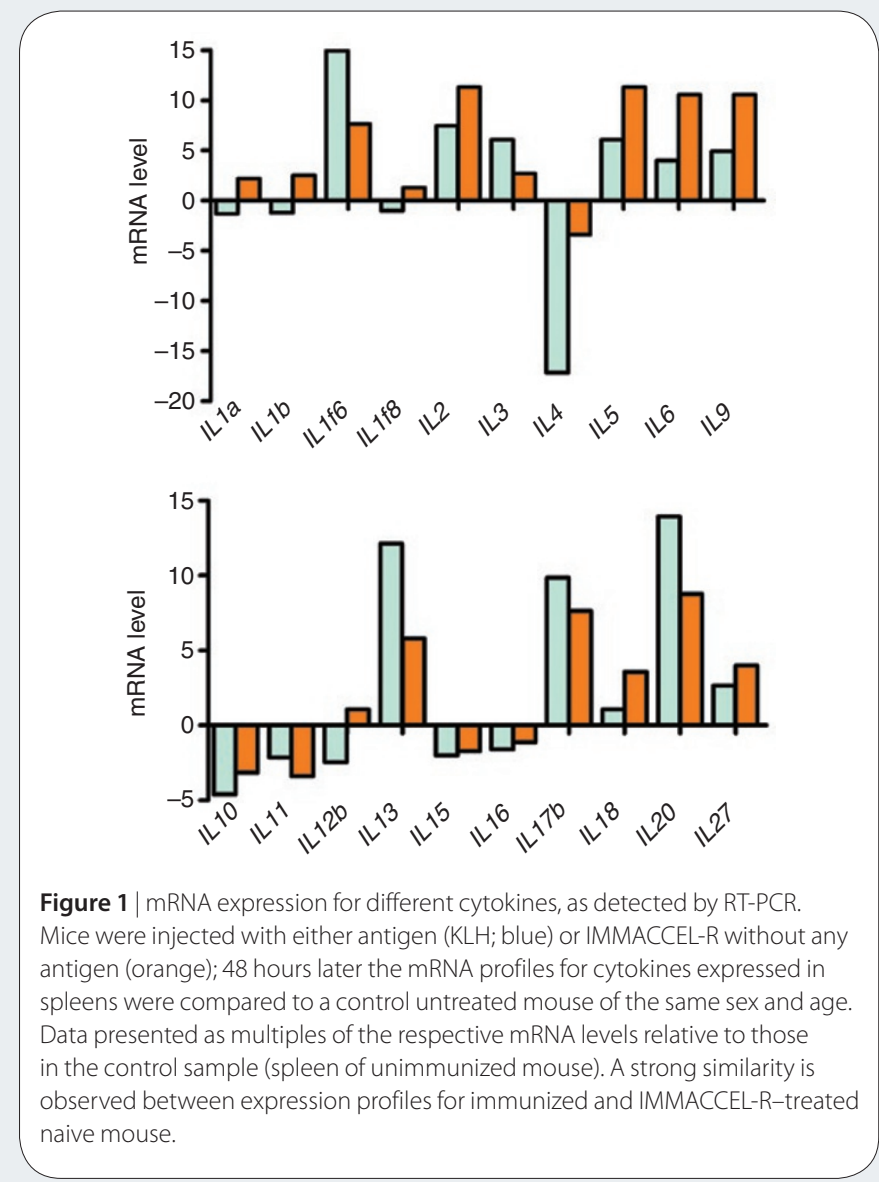



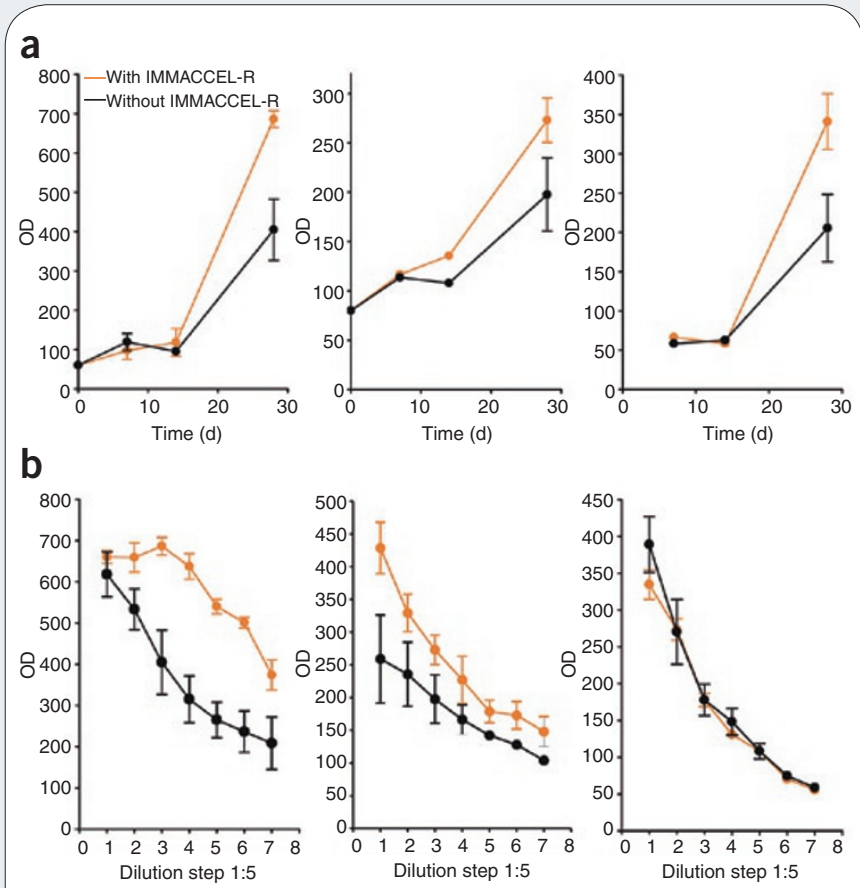

Figure $\mathbf{2} \mid$ Antibody production. (a) Sera from mice that had been injected once with DNP-KLH, with or without IMMACCEL-R, were collected at days 0, 7, 14 and 28 and analyzed for the presence of antigen-specific antibodies of a certain isotype. (b) The relative titer of specific antibodies was analyzed by ELISA for samples from day 28. Data are shown as mean + s.d.; three mice in every group.

antigen. Owing to IMMACCEL-R's effect on immune response, earlier and stronger antibody responses are observed, as well as earlier switch of specific antibody from immunoglobulin $M(\lg M)$ to $\lg G 1$ and $\lg G 2$ isotypes. By day 28, the titer of specific antibodies is already up to 30 times higher for IgGs of $\gamma 1$ and $\gamma 2$ a isotypes, and 2-4 times lower for IgM (Fig. 2). The adjuvant works equally well for all mammalian species tested (mouse, rabbit, goat and swine). A slightly changed version of the molecule is also effective in birds (chicken). The adjuvant is nontoxic and works with all tested types of antigens: proteins, glycoproteins, peptides and haptens conjugated to a carrier.

\section{Use for development of antibodies}

Unique adjuvant properties of IMMACCEL-R allowed PickCell to develop an accelerated protocol for production of specific antisera in rabbits. Immunization with IMMACCEL-R achieves, within 28 days, specific-antibody IgG titers in rabbits similar to those obtained with a standard 80-day protocol. When prepared against strong peptide antigen, the affinity-purified antibodies can be used in many applications and do not differ in affinity or specificity from those obtained through a standard immunization scheme (Fig. 3). We also tested the adjuvant for immunizing mice for hybridoma development. Mice were injected with E7 recombinant protein of HPV16 or HPV18 in a mix with

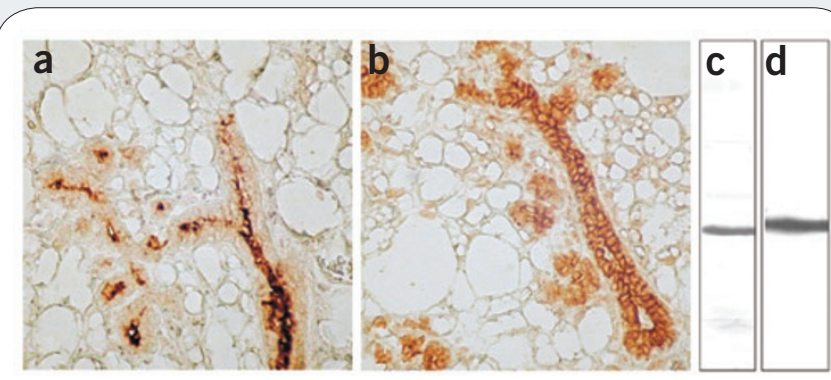

Figure 3 | Quality of sera prepared using IMMACCEL-R in 28-day immunization scheme. (a,b) Immunohistochemistry analysis of formalin-fixed sections of mouse mammary gland using antibodies to whey acidic protein (a) and mouse cytokeratin-18 (b). (c,d) Immunoblotting analysis of mouse mammary tumor cells using antibodies to mouse cytokeratin-18 (c) and mouse cytokeratin-19 (d).

IMMACCEL-V, and their spleens were used for fusion on day 10 after booster immunization on day 7 . About $20 \%$ of all isolated clones were positive for specific antibodies to the protein used for immunization, with over $90 \%$ of clones producing antibodies of the IgG isotype.

\section{Further development}

As effective humoral immune response requires the presence in antigen of active T-helper epitopes and effective delivery of antigen to dendritic cells, we produced a new carrier molecule with adjuvant properties that combines dendritic cell-targeting sequences with effective T-helper promiscuous epitopes and molecules increasing the antigen presentation by dendritic cells. This new adjuvant, called MAD ${ }^{\mathrm{TM}}$, is especially effective in inducing a strong immune response to weakly immunogenic recombinant proteins and works with both standard and accelerated protocols, always providing higher titers of specific antibody. Thus, for small fusion proteins (15-30 kDa), using MAD we obtain, on average, 4-20 times higher titer while using 4 times less antigen than in immunizations without MAD.

\section{Conclusions}

Using new adjuvants from PickCell strongly accelerates the response, increases the specific antibody titer and enhances immunogenicity of poor antigens, all while using substantially less protein. This technology, already tested and used in thousands of custom antibody projects performed by PickCell, may be highly useful to all groups producing antibodies or hybridomas. Additional product information is available at PickCell's website, http://www.pickcell-labs.com/.

1. Kieber-Emmons, T. et al. Therapeutic peptides and peptidomimetics. Curr. Opin. Biotechnol. 8, 435-441 (1997).

2. Litvinov, S.V. Peptides for immunoboosting. International patent application WO 2007/110722A8 (2007).

This article was submitted to Nature Methods by a commercial organization and has not been peer reviewed. Nature Methods takes no responsibility for the accuracy or otherwise of the information provided. 